\title{
Cambios de usos de suelo y vegetación en la comunidad agraria Kashaama, Anzoátegui, Venezuela: 2001-2013
}

\section{Changes in land use and vegetation in the agrarian community Kashaama, Anzoátegui, Venezuela: 2001-2013}

\author{
Barlin Orlando Olivares-Campos ${ }^{1}$ \\ Universidad de Córdoba, España \\ Miguel Armando López-Beltrán ${ }^{2}$ \\ Universidad Autónoma de Sinaloa, México \\ Deyanira Lobo-Luján \\ Universidad Central de Venezuela, Venezuela
}

\section{Resumen}

Los cambios en el uso del suelo y vegetación que soporta la tierra son cada vez más relevantes para poder comprender los elementos de roce entre las comunidades agrícolas y los ecosistemas terrestres que les sirven de soporte. El objetivo de este estudio fue analizar los cambios de usos de suelo y vegetación en la comunidad indígena Kashaama, Estado Anzoátegui, Venezuela. Se utilizaron imágenes digitales derivadas de un espectroradiómetro para imágenes de resolución moderada (Moderate Resolution Imaging Spectroradiometer - MODIS) que contienen información sobre las diferentes coberturas de uso de la tierra y vegetación, para los años 2001 y 2013. La comunidad indígena estudiada presenta un proceso significativo de transformación de la cobertura vegetal a usos antrópicos del suelo, poniendo en riesgo los bienes y servicios ecosistémicos para la población.

Palabras clave: agricultura, degradación, geomática, sustentabilidad, ecosistema.

1 Doctorando. Universidad de Córdoba. Programa de Doctorado en Ingeniería Agraria, Alimentaria, Forestal y del Desarrollo Rural Sostenible. España. Teléfono: +58 4243173568. E-mail: barlinolivares@gmail.com.

2 Universidad Autónoma de Sinaloa, Facultad de Agronomía, México. E-mail: miguel.armandolb@gmail.com

3 Universidad Central de Venezuela, Facultad de Agronomía. Maracay estado Aragua. E-mail: lobo.deyanira@gmail.com 


\begin{abstract}
Changes in land use and vegetation sustained by planet earth are evermore so relevant in understanding the elements of friction between agricultural communities and terrestrial ecosystems that sustain them. The objective of this study was to analyze changes in land use and vegetation in the indigenous community of Kashaama, Anzoátegui State, Venezuela. Digital images derived from a moderate resolution imaging spectroradiometer (MODIS) were used that contain information on the different land use and vegetation coverage for the years 2001 through 2013. The studied indigenous community presents a significant transformation process from vegetation coverage to anthropic land use, putting at risk the ecosystem's goods and services for the population.
\end{abstract}

Keyword: Agriculture; degradation; geomatics; sustainability: ecosystem.

\title{
Introducción
}

Básicamente, las actividades humanas tales como el desarrollo de cultivos y áreas extensas de pastizales han cambiado drásticamente la cobertura del planeta. Hace décadas se evidenció que los cambios de cobertura y de uso de suelo influyen directamente en el ciclo hidrológico, la pérdida de biodiversidad, la erosión de los suelos y el aumento de diversos gases incrementan el efecto invernadero. Estos cambios en el uso de suelo han permitido al ser humano apropiarse de los recursos, pero al mismo tiempo han restringido la capacidad de los ecosistemas en regenerarse y poder sostener la demanda de producción de alimentos y los recursos forestales, no existe una regulación en el clima, afectando la disponibilidad del agua y la calidad del aire (Foley, 2005; FAO, 2008; García-Mora y Mas, 2011).

En Venezuela, las tierras de uso agrícola están severamente degradadas debido a un uso excesivo de maquinarias e insumos químicos, entre otros aspectos. Así mismo, las necesidades crecientes de tierras para desarrollos urbanos e industriales se cubren muchas veces a costa de pérdidas de tierra de alta capacidad agrícola, generando importantes problemas de degradación de los suelos (Lozano et al., 2002; Comerma y Chacón, 2002; Rodríguez y Rey, 2004; Lugo-Morin, 2007).

De acuerdo con la investigación desarrollada por Comerma y Paredes (1978) se establece que las áreas sin limitaciones para el desarrollo agrícola en Venezuela ocupan apenas el $2 \%$ del territorio, la mayoría de las áreas tienen limitaciones de relieve (44\%) y de baja fertilidad (32\%), de mal drenaje (18\%) y aridez o escasez de lluvia (4\%). Justamente, esta condición general del país hace que la actividad agrícola, si no es bien 
llevada bajo preceptos de sostenibilidad, puede generar o intensificar los procesos de degradación que influyen en la calidad de los suelos.

Más allá de esta problemática ambiental, la degradación de suelos en la región conlleva agudos problemas sociales y económicos, como la pérdida de rentabilidad vinculada a la degradación de los recursos naturales, presentando un escenario adverso a los agricultores de las comunidades indígenas, quienes se ven en la obligación de migrar forzosamente al desempleo por la improductividad de las tierras; por otro lado, los agricultores que se quedan frente a predios agropecuarios degradados, enfrentan la carencia de alimentos.

Esta problemática se ha visto reflejada en la mayoría de las tierras orientales venezolanas, debido, principalmente, a las operaciones de exploración y producción de crudos de las empresas petroleras que operan en el municipio Freites de Anzoategui, desafectando cientos de hectáreas, causando daños serios al medio ambiente, en la mayoría de los casos irrecuperables.

La evaluación de la cobertura del suelo representa una parte fundamental del proceso de planificación del uso de la tierra, considerado como un trabajo complejo, dinámico y transdisciplinario, donde se presentan las siguientes acepciones: el reconocimiento de la necesidad de un cambio, la identificación de objetivos, la formulación de propuestas para implementar formas alternativas de uso, manejo e identificación de necesidades, el reconocimiento y la delimitación de los diferentes usos de suelo, entre otros aspectos (Salas et al., 2008; Comerma, 2002; Delgado, 2007).

El objetivo de este estudio fue analizar la evolución del uso de suelo y vegetación en la comunidad indígena Kashaama, Estado Anzoátegui, mediante imágenes derivadas de un espectroradiómetro para imágenes de resolución moderada (Moderate Resolution Imaging Spectroradiometer - MODIS).

\section{Marco metodológico}

\section{a. Definición y selección del área de evaluación}

La comunidad indígena Kari'ña de Kashaama está ubicada en la parroquia Cantaura del municipio Pedro María Freites, perteneciente a la región de los llanos orientales venezolanos, a una distancia de $165 \mathrm{~km}$ de Barcelona, capital del Estado Anzoátegui. Posee una extensión territorial 
de aproximadamente 5272 ha y actualmente cuenta con una población de 1392 habitantes (Olivares et al., 2015). La delimitación del área total de la comunidad estuvo en función de los ejercicios de mapeo y las entrevistas con los informantes claves de la comunidad (Figura 1).

Figura 1. Geolocalización de la comunidad indígena de Kashaama en el estado Anzoátegui, Venezuela.

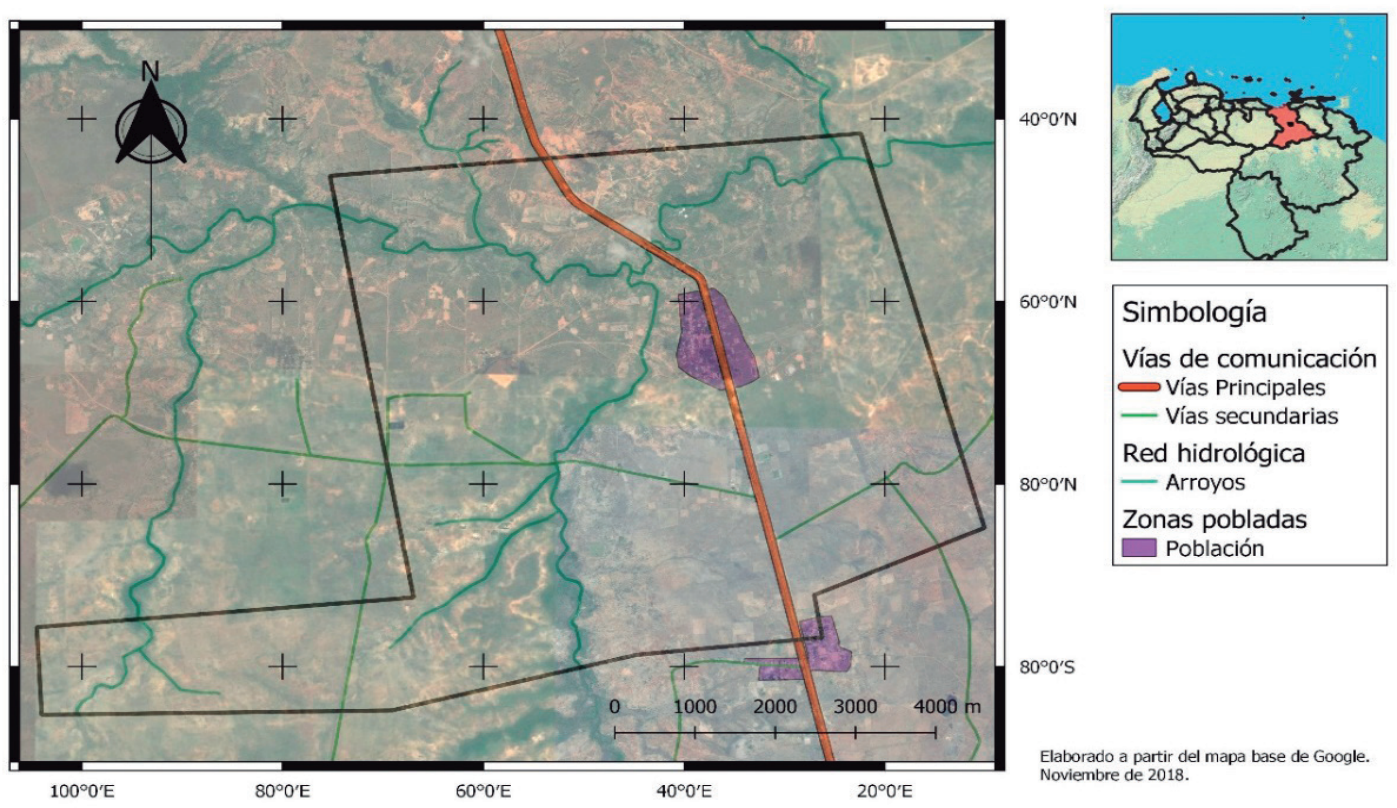

Fuente: elaboración propia.

La precipitación en la comunidad de Kashaama se presenta con una estación seca bien definida de diciembre a abril, con un promedio de precipitación anual (1970-2000) en la región de $1119 \mathrm{~mm}$ (Olivares et al., 2013b). La región presenta un bioclima seco tropical, según la clasificación de Holdridge, con evaporación media anual de $2626 \mathrm{~mm}$; la temperatura media anual de $26,9^{\circ} \mathrm{C}$ y la humedad relativa media anual de $78 \%$ (Olivares et al., 2015; Olivares et al., 2017c). 


\section{b. Evaluación de la cobertura y uso}

En este estudio, se utilizó un producto enfocado al estudio de las cubiertas terrestres (MODLAND) obtenidos con el satélite Terra. El producto MCD12Q1 es una imagen digital que contiene información sobre las diferentes coberturas de uso de suelo y vegetación proporcionado por el sensor MODIS, brinda información en base a diferentes sistemas clasificatorios. Este producto es la combinación de datos de dos plataformas (TERRA y AQUA) de MODIS. El resultado final está basado en una clasificación supervisada, obtenida con árboles de decisión cambiando la distribución en áreas de entrenamiento. Utiliza como insumo la distribución de la reflectancia bidireccional, índice de vegetación mejorado (EVI) y la temperatura superficial (García-Mora y Mas, 2011).

Se descargaron los archivos para el año 2001 y 2013, por ser el periodo de tiempo lo suficientemente largo y contrastante para fines de la evolución del uso del suelo. Adicionalmente, este periodo de tiempo se ajusta a los lineamientos e intereses del proyecto de investigación e innovación tecnológica descrito por Olivares et al., (2016a). La decisión de realizar dicho estudio en esta comunidad responde a la condición de vulnerabilidad agrícola de la comunidad Kashaama (Olivares et al., 2017a), además de ser una comunidad indígena típica de la etnia Kari'ña. La información correspondiente del producto se muestra en el Cuadro 1. Se seleccionó el sistema de clasificación IGBP (Programa Internacional Geosfera - Biosfera) compuesta por 18 categorías. (cuadro 2)

El procesamiento se realizó con el software de Sistema de Información Geográfica IDRISI. Usando el módulo extract se obtuvieron los valores estadísticos descriptivos para cada uso de suelo y vegetación para cada año de estudio. Posteriormente, se realizó un análisis comparativo para determinar los cambios que ocurrieron en comparación de ambos años. Para este procesado se utilizó el Land Change Modeler integrado en el SIG.

Para facilitar el manejo de la información se realizó una reclasificación de las distintas categorías quedando de la siguiente manera: 0. Agua; 1. Bosques; 2. Matorral; 3. Sabanas; 4. Pastos; 5. Humedales permanentes; 6. Cultivos; 7. Áreas urbanas; 8. Mosaico de cultivos y vegetación natural; 9. Suelo desnudo o con escasa vegetación. (cuadro 2) 
Barlin Orlando Olivares-Campos - Miguel Armando López-Beltrán - Deyanira Lobo-Luján Changes in land use and vegetation in the agrarian community Kashaama, Anzoátegui, Venezuela: 2001-2013

Cuadro 1. Características del producto MCD12Q1.

\begin{tabular}{cc}
\hline Características & Descripción \\
\hline Resolución espacial & $500 \mathrm{~m}$ \\
\hline Resolución temporal & Anual \\
\hline Proyección & Sinusoidal \\
\hline $\begin{array}{c}\text { Sistemas de } \\
\text { clasificación }\end{array}$ & Esquema de clasificación del Programa Internacional de \\
& Geosfera - Biosfera (IGBP) \\
& Esquema de clasificación de la Universidad de Maryland \\
& diación absorbida fotosintéticamente activa \\
& Esquema de producción primaria neta \\
& Esquema de tipo de función vegetativa \\
\hline
\end{tabular}

Fuente: adaptado de LP DAAC, 2014; García-Mora y Mas, 2011 a.

El procesamiento se realizó con el software de Sistema de Información Geográfica IDRISI. Usando el módulo extract se obtuvieron los valores estadísticos descriptivos para cada uso de suelo y vegetación para cada año de estudio. Posteriormente, se realizó un análisis comparativo para determinar los cambios que ocurrieron en comparación de ambos años. Para este procesado se utilizó el Land Change Modeler integrado en el SIG.

Para facilitar el manejo de la información se realizó una reclasificación de las distintas categorías quedando de la siguiente manera: 0. Agua; 1. Bosques; 2. Matorral; 3. Sabanas; 4. Pastos; 5. Humedales permanentes; 6. Cultivos; 7. Áreas urbanas; 8. Mosaico de cultivos y vegetación natural; 9. Suelo desnudo o con escasa vegetación.

Cuadro 2. Esquema de clasificación del Programa Internacional de Geosfera - Biosfera (IGBP).

\section{Códigos de las características}

\section{Agua}

1. Bosques perennes de coníferas

2. Bosque perennes de latifoliadas

3. Bosque deciduo de coníferas
6. Matorral cerrado

7. Matorral abierto

8. Sabanas arboladas

9. Sabanas
12. Cultivos

13. Áreas urbanas y construidas

14. Mosaico de cultivos y vegetación natural

15. Nieve y hielo 


\section{Códigos de las características}

4. Bosque deciduo de latifoliadas

5. Bosques mixtos
10. Pastos

11. Humedales permanentes
16. Suelo desnudo o con escasa vegetación

Fuente: adaptado de García-Mora y Mas, 2011b.

En el análisis y cuantificación de los cambios de uso de suelo y vegetación se utilizó la ecuación 1 (FAO, 1996), para el cálculo de la Tasa de Cambio Anual (TCA):

$$
\mathrm{TCA}=\left[\frac{\mathrm{S}_{2}}{\mathrm{~S}_{1}}\right]^{1 / \mathrm{n}}-1 * 100
$$

Donde, (S2): superficie fecha más tardía; (S1): superficie en la fecha más temprana; (n): es el número de años entre las dos fechas; expresarlo en porcentaje.

\section{c. Planteamientos acerca de alternativas del Manejo Sostenible de la Tierra}

Para describir los planteamientos de los componentes del Manejo Sostenible de la Tierra (MST) en la zona de estudio, se utilizó el modelo basado en fuerzas motrices, presión, estado, impacto y respuesta (FPEIR), desarrollado por la Organización para la Cooperación y el Desarrollo Económicos (OECD, 1993), el cual ha sido adoptado por FAO/LADA (2009) y Urquiza et al. (2011) para la evaluación de la degradación de la tierra y la identificación de puntos de intervención del MST.

Esta metodología constituyó una herramienta para identificar las opciones de manejo del conjunto de problemas ambientales detectados en la comunidad indígena. El modelo capturó las fuerzas determinantes y las presiones - esencialmente controladas por la actividad humana- y sus efectos sobre el sistema ambiental y el estado de los recursos naturales (Banco Mundial, 2008).

\section{Resultados y discusión}

\section{a. Evaluación de la cobertura y uso de la tierra}

El análisis del uso de suelos y vegetación muestra que la mayor TCA negativa se presentó para el Matorral, siendo esta la más impactada con un cambio neto negativo de 375.7 ha. Por su parte, la categoría de mosaicos 
de cultivos y vegetación natural presenta la mayor TCA positiva que el resto de las categorías con 994.53 ha (Cuadro 3).

La clasificación de las categorías de MCD12Q1 se basa en la cobertura total del pixel, siempre y cuando sea mayor al $60 \%$ de cobertura en el área, asigna su clase acorde a los tipos de clasificación. En el caso de los mosaicos, es una clase combinada que integra de un $40-60 \%$ a zonas cultivables en combinación de árboles, arbustos o vegetación herbácea, representando una categoría denominada mosaico de cultivos y vegetación natural (Sulla-Menashe y Friedl, 2018).

Cuadro 3. Comparación de la cobertura de uso del suelo y vegetación en la comunidad indígena de Kashaama, Anzoátegui.

\begin{tabular}{lccc}
\hline \multirow{2}{*}{ Categoría } & \multicolumn{2}{c}{ Hectáreas } & \multirow{2}{*}{ TCA (\%) } \\
\cline { 2 - 3 } \multicolumn{1}{c}{ Año 2001 } & Año 2013 & \\
\hline Matorral & 397.81 & 22.11 & -23.10 \\
Sabanas & 1127.14 & 1524.95 & 2.79 \\
Pastos & 3514.02 & 2585.79 & 2.74 \\
Cultivos & 154.71 & 66.31 & 7.41 \\
Áreas urbanas y construidas & 44.21 & 44.21 & 0 \\
Mosaicos de cultivos y & 88.41 & 1082.94 & 25.57 \\
vegetación natural & & & \\
\hline
\end{tabular}

Fuente: elaboración propia.

La Figuras 2 y 3 muestran la cobertura espacial de las categorías del uso de suelo y vegetación en la zona de estudio para los dos años de referencia. A nivel regional (zonas agrícolas indígenas de Anzoátegui) los cambios de uso y cobertura podrían provocar modificaciones en los ciclos hídricos, alteraciones en los regímenes de temperatura y precipitación, favoreciendo con ello el calentamiento global, la disminución en el secuestro de bióxido de carbono y la pérdida de hábitats y biodiversidad local de los llanos orientales venezolanos.

La disminución de la cobertura vegetal tiene implicaciones que inciden en la disponibilidad y calidad de los bienes y servicios ambientales que ofrecen estas tierras. Entre otros, podría afectar la infiltración del agua de lluvia, ya que a pesar de ser suelos principalmente arenosos serían susceptibles al sellado y encostrado (Lozano et al., 2002); en consecuencia, se afectaría la recarga de los mantos hídricos y el equilibrio en el ciclo del 
Barlin Orlando Olivares-Campos - Miguel Armando López-Beltrán - Deyanira Lobo-Luján

Cambios de usos de suelo y vegetación en la comunidad agraria Kashaama, Anzoátegui, Venezuela: 2001-2013

agua, considerado esto como un aspecto de importancia en este estado oriental del país, debido al potencial de los acuíferos.

Figura 2. Mapa de uso de suelo y vegetación en Kashaama. Año 2001.
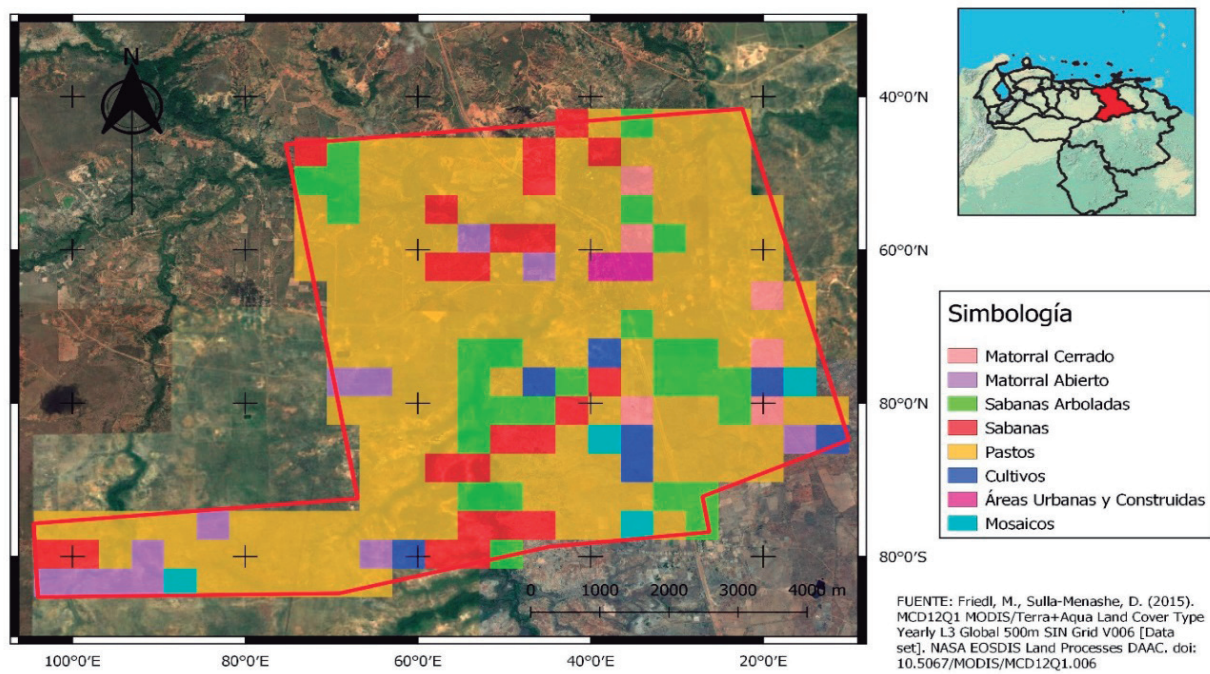

$60^{\circ} \mathrm{O}^{\prime} \mathrm{N}$

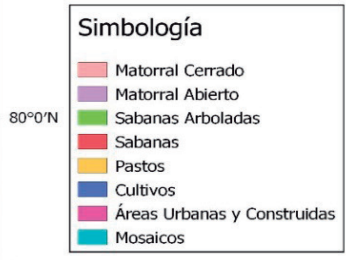

$80^{\circ} 0^{\prime} \mathrm{S}$

FUENTE: Friedl, M., Sulla-Menashe, D. (2015). MCD12Q1 MODIS/Terra+Aqua Land Cover Type Yearly L Global 500m SIN Grid V006 [Data 10.5067/MODIS/MCD1201.006

Fuente: elaboración propia.

Figura 3. Mapa de uso de suelo y vegetación en Kashaama. Año 2013.
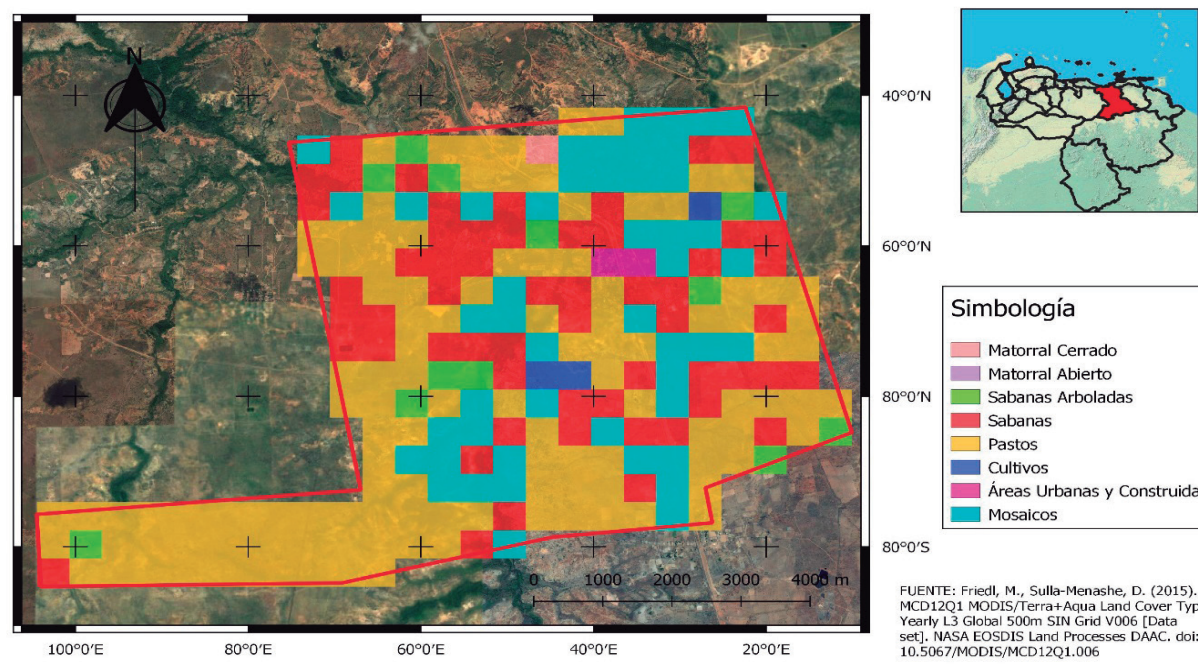

$60^{\circ} 0^{\prime} \mathrm{N}$

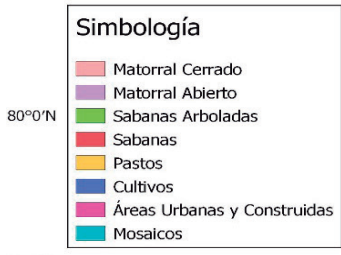

$80^{\circ} 0^{\prime} 5$

FUENTE: Friedl, M., Sulla-Menashe, D. (2015). Yeatl L1 GoDis/Terra+Aqua Land Cover Type 5et]. NASA EOSDIS Land Processes DAAC, do: 10.5067/MODIS/MCD 12Q1.006

Fuente: elaboración propia. 
La ganancia y pérdida para cada categoría de cobertura (Figura 4) permite interpretar de manera general los cambios de la cobertura durante el periodo evaluado. A título ilustrativo, se observa que la categoría correspondiente a pastos, que además presenta el mayor cambio total, muestra casi un $40 \%$ de pérdidas (1612 ha) y cerca de $25 \%$ de ganancias (620 ha). Algunas categorías muestran principalmente ganancias, por ejemplo, las que representan los mosaicos de vegetación natural y cultivos seguida de las sabanas. Es de resaltar que, los asentamientos humanos o áreas urbanas se mantuvieron sin cambio alguno.

Figura 4. Pérdidas y ganancias de la cobertura del suelo para los años 2001 y 2013 en Kashaama.

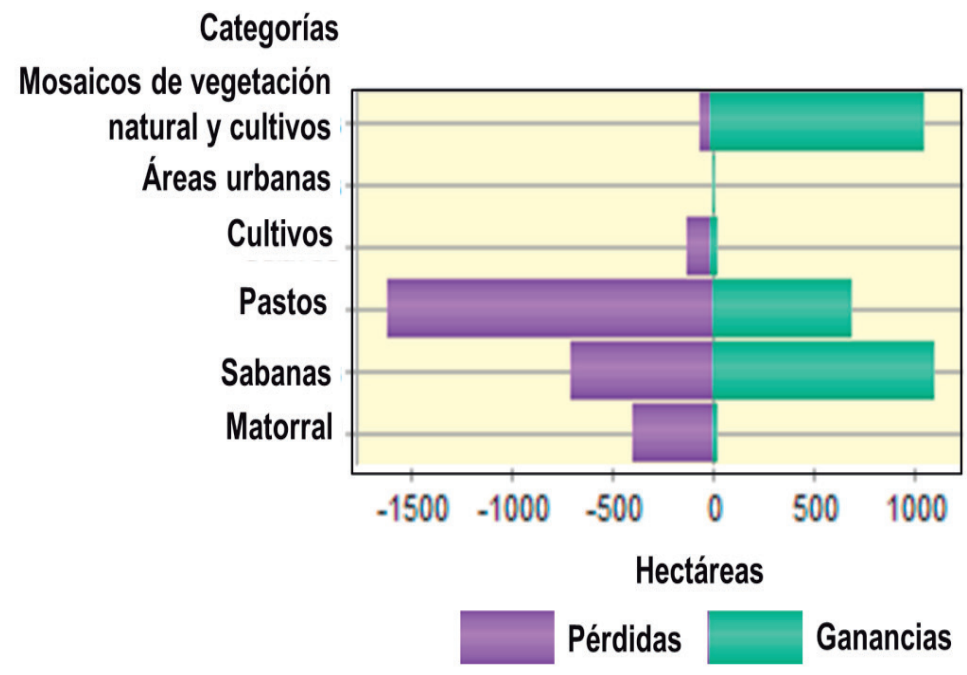

Fuente: elaboración propia.

El Cuadro 4 muestra las zonas persistentes en el área de estudio. El uso actual de la tierra está comprendido en urbano y agrícola, en la zona se presentan diversos sistemas de explotación (siembra en conuco y siembra en hileras), caracterizados por la presencia de Yuca (Manihot esculenta Crantz), Maní (Arachis hypogaea), Fríjol (Vigna sinensis L.), Patilla (Citrullus vulgaris), Melón (Cucumis melo L.), Maíz (Zea mays L.), Sorgo (Sorghum bicolor Moench) y pastizales con especies introducidas 
(Brachiaria brizantha, B. dictyoneura, B. decumbens y B. humidicola) (Olivares et al., 2016b).

Cuadro 4. Zonas persistentes en el área de Kashaama.

\begin{tabular}{cc}
\hline Categoría & Hectáreas \\
\hline Matorral & 0 \\
Sabanas & 419.92 \\
Pastos & 1900.66 \\
Cultivos & 22.10 \\
Áreas urbanas y construidas & 44.21 \\
Mosaicos de cultivos y vegetación natural & 22.10 \\
\hline
\end{tabular}

Fuente: elaboración propia.

Las unidades de tierras estudiadas se caracterizan por presentar perfiles con una marcada acidez en cada uno de los horizontes descritos, así como un grado de fertilidad bajo. Hay presencia predominante, en la mayoría de los casos, de textura arenosa en la parte superior del perfil, razón por la cual los suelos tienen infiltración y percolación rápida; la retención de humedad en la zona radicular es baja. Los horizontes presentan muy bajo contenido de materia orgánica, de allí la vegetación predominante representada por pastos.

Los suelos predominantes del área son Ultisoles y Oxisoles (Quartzipsamments, Kandiustults, Haplustox) y presentan un avanzado desarrollo pedogenético con la excepción de aquellos formados por un esqueleto de arenas de cuarzo en su mayoría Entisoles, donde no ha podido desarrollarse un proceso pedogenético, debido a la resistencia que presentan estos materiales, coincidiendo con Zinck y Urriola (1970)

De acuerdo al estudio desarrollado por Olivares (2012a), los suelos representativos de la zona en estudio presentan valores de $\mathrm{pH}$ moderadamente ácido $(<5,8)$, con contenidos bajos a muy bajos de fósforo $(\sim 6 \mathrm{mg}$. $\mathrm{kg}-1)$, calcio ( $35 \mathrm{mg} . \mathrm{kg}-1)$, magnesio $(\sim 37 \mathrm{mg}$. kg-1), potasio (10-30 $\mu \mathrm{g} / \mathrm{g})$; contenidos variables de hierro (0,5-30 mg. kg-1), manganeso (0,512 mg. kg-1), cinc (0,1-1 mg. kg-1), bajo contenido de materia orgánica $(\sim 0,51 \%)$, bajo a medio de aluminio intercambiable $(\sim 0,44 \mathrm{cmol} \mathrm{kg}-1)$ y baja conductividad eléctrica $(\sim 0,28 \mathrm{dS}$. m-1). 
Los resultados obtenidos (Cuadro 5) indican que la dinámica de cambio es mayor sobre las coberturas vegetales (Pastos), las cuales cambian a otros usos de suelo (Mosaicos de vegetación natural y cultivos). Los cambios ocurridos en los distintos paisajes naturales y socioculturales que comprenden a la comunidad indígena de Kashaama están relacionados, principalmente, con la disminución y pérdida de los ecosistemas terrestres de sabana (Figura 5). Los resultados presentados muestran que efectivamente este territorio ha experimentado un proceso de deforestación en un período de 12 años (Figura 6).

Cuadro 5. Transición de la cobertura y uso del suelo durante 2001 y 2013 en Kashaama.

\begin{tabular}{lc}
\hline \multicolumn{1}{c}{ Categorías } & Hectáreas \\
\hline Sabanas a Matorral & 22.10 \\
Matorral a Sabanas & 44.20 \\
Pastos a Sabanas & 972.43 \\
Cultivos a Sabanas & 66.30 \\
Mosaicos de vegetación natural y cultivos a Sabanas & 22.10 \\
Matorral a Pastos & 309.41 \\
Sabanas a Pastos & 287.31 \\
Cultivos a Pastos & 44.20 \\
Mosaicos de vegetación natural y cultivos a Pastos & 44.20 \\
Sabanas a Cultivos & 22.10 \\
Pastos a Cultivos & 22.10 \\
Matorral a Mosaicos de vegetación natural y cultivos & 44.20 \\
Sabanas a Mosaicos de vegetación natural y cultivos & 375.71 \\
Pastos a Mosaicos de vegetación natural y cultivos & 618.82 \\
Cultivos a Mosaicos de vegetación natural y cultivos & 22.10 \\
\hline
\end{tabular}

Fuente: elaboración propia. 
Barlin Orlando Olivares-Campos - Miguel Armando López-Beltrán - Deyanira Lobo-Luján

Cambios de usos de suelo y vegetación en la comunidad agraria Kashaama, Anzoátegui, Venezuela: 2001-2013

Figura 5. Mapa sin cambios de coberturas durante el periodo (2001 y 2013) en Kashaama.
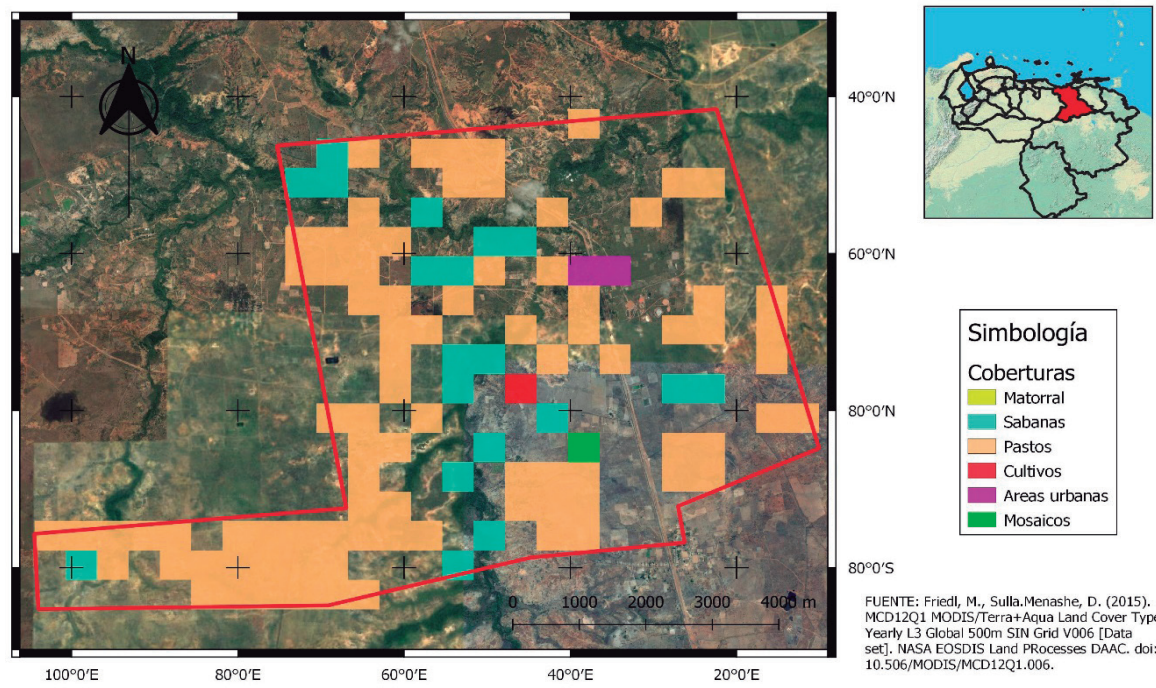

$60^{\circ} 0^{\prime} \mathrm{N}$

\begin{tabular}{|l|}
\hline Simbología \\
Coberturas \\
Matorral \\
Sabanas \\
Pastos \\
Cultivos \\
Areas urbanas \\
Mosaicos \\
\hline
\end{tabular}

$80^{\circ} 0^{\prime S}$

FUENTE; Friedl, M., Sulla.Menashe, D. (2015). MCD 12Q1 MODIS/Terra+Aqua Land Cover Type Yearly L3 Global $500 \mathrm{~m}$ SIN Grid v006 [Data 10.506/MODIS/MCD12Q1.006.

Fuente: elaboración propia.

Figura 6. Mapa de transición de uso de suelo y vegetación entre el año 2001 y 2013 en Kashaama.
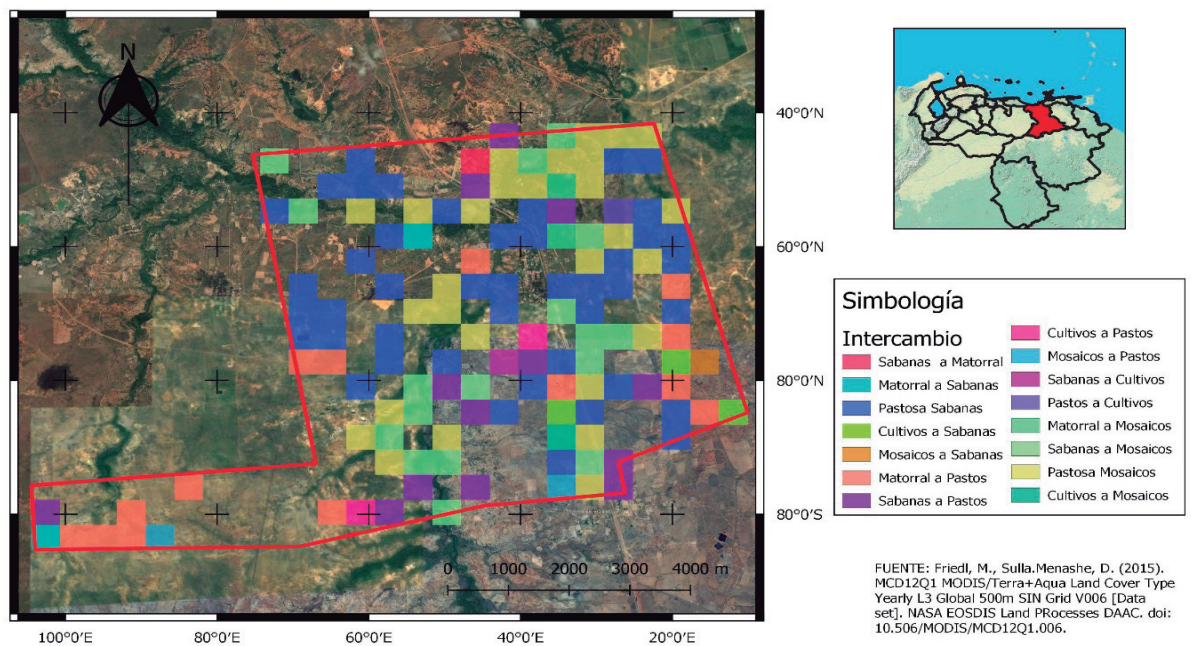

FUENTE: Friedl, M., Sulla.Menashe, D. (2015). Yearly 13 Glohal $500 m$ SIN Grid Vo06 [Data set]. NASA EOSDIS Land PRocesses DAAC, doi: 10.506/MODIS/MCD12Q1.006.

Fuente: elaboración propia. 
Un cambio en la cobertura de sabanas a matorral debido a una decisión en el manejo de la tierra es capaz de cambiar desde un nivel local a regional la captación de agua en los suelos, además de que el cambio en la vegetación puede afectar los regímenes de precipitación (Rodríguez et al., 2013) y temperatura (Olivares et al., 2013a). Por otro lado, el calentamiento global modifica directamente la localización y distribución de los diferentes tipos de cobertura de suelo, en particular por su impacto en los ecotonos (zonas de transición), o de manera indirecta por un aumento en la ocurrencia de incendios forestales (Colditz et al., 2014a).

Según Olivares et al. (2017b), la agricultura que se practicaba anteriormente en Kashaama fue el resultado del desarrollo de diversas estrategias adaptativas y de la práctica tradicional, basada en criterios de conservación de los recursos naturales (ríos, tierra, vegetación natural, fauna, y aire). Estos conocimientos, según explicaron, constituyeron el conocimiento del medio natural de importante valoración por los indígenas a lo largo del tiempo.

La diversidad de los cultivos practicados por los Kari'ña ha sido ampliamente estudiada por investigadores como Olivares y Franco (2015) y Olivares et al. (2012), quienes establecieron un abanico de plantas comúnmente utilizados por la etnia Kari'ña, donde solo las raíces y tubérculos, tales como: yuca amarga, yuca dulce, batata junto con el plátano (Musa $\mathrm{AAB})$ fueron considerados como básicos en su alimentación.

Es común observar en la comunidad, la distancia considerable que existe entre los conucos cercanos al Río Cachama y las viviendas de los productores indígenas. Debido a esto, una estrategia que han desarrollado varias familias en el sector ha sido el establecimiento de huertos familiares o patios productivos en el terreno de las viviendas.

Hoy en día, este tipo de sistema de producción es considerado como modelo agrícola en los planes nacionales, por sus antecedentes de garantizar la seguridad alimentaria de comunidades rurales en el pasado. Como se mencionó anteriormente, la dieta de los indígenas Kari'ña ha sufrido ciertas transformaciones, al igual que ciertos aspectos sociales y culturales, el principal motivo de estos cambios estuvo asociado al abandono o descuido de las actividades agrícolas como principal actividad de subsistencia; a esto se le sumó la carencia de los servicios básicos en el sector y la apatía 
de algunos miembros por no tener acceso a créditos, insumos, servicios agrícolas o planes de capacitación.

En Kashaama la agricultura se ha visto afectada como consecuencia directa o indirecta del deterioro ambiental ocasionado por las actividades petroleras, el acaparamiento de tierras por la industria de hidrocarburos y las limitaciones en cuanto al acceso de créditos agrícolas (Olivares y Franco, 2015). Esto, de alguna manera, ha generado que muchos Kari'ña se hayan visto obligados a trabajar como asalariados en empleos fijos u ocasionales (obreros de la construcción, mano de obra no especializada, labores domésticas y agrícolas). Así, se ha verificado que estas migraciones tienen una base socioeconómica y a su vez generan una fuerte desarticulación social que amenaza la reproducción cultural Kari'ña (Olivares, 2012b; 2014). Por ejemplo, es frecuente que algunas comunidades estén habitadas solo por ancianos y niños, los adultos y jóvenes económicamente activos tienen que salir hacia los centros urbanos en busca de trabajo asalariado.

\section{b. Susceptibilidad de las tierras a la degradación}

Los suelos del área de estudio presentan grandes limitaciones de productividad, especialmente desde el punto de vista de fertilidad química para la producción de cultivos y forrajes. Asimismo, el riesgo de erosión hídrica, considerando la tolerancia de pérdida de suelo en función de la profundidad efectiva de los suelos ha resultado moderadamente bajo, estimado para un importante grupo de tipos de utilización de la tierra (TUT), recomendando prácticas conservacionistas para aquellos TUT que ofrezcan poca cobertura al suelo (Ferrer y Comerma, 1997). Este aspecto es de suma importancia, ya que la distribución de las lluvias es estacional y la zona presenta muy alta agresividad climática (Lobo et al., 2010).

La escasa cobertura del suelo que producen los cambios de uso de la tierra no solo conlleva a bajos aportes de materia orgánica a los suelos, la cual es mineralizada rápidamente, sino que ofrece insuficiente protección a los suelos contra los procesos erosivos, como se observan en la Figura 7. Estas cárcavas, generalmente son producto de la erosión regresiva, como las describieron Rojas (1981); Olivares y Cortez (2017). 
Figura 7. Ilustraciones de las cárcavas de la consecuencia de la erosión hídrica en Kashaama.
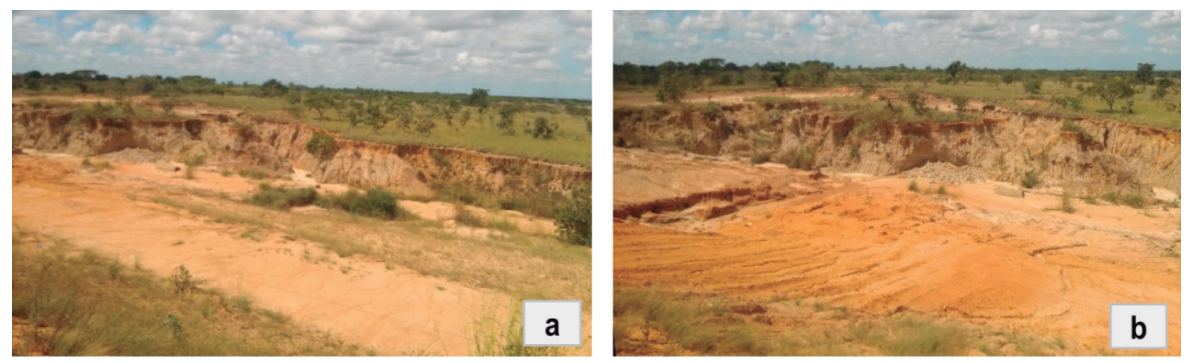

Fuente: elaboración propia.

\section{c. Planteamientos para el Manejo Sostenible de la Tierra (MST)}

La degradación de la tierra da como resultado la pérdida de servicios ecosistémicos, lo cual lesiona adicionalmente la sostenibilidad de los ecosistemas tanto administrados como naturales. Se evidencia que en la comunidad indígena Kashaama, la degradación de la tierra no es intencional sino indirecta; y resulta de la falta de cuidado o de la inevitable lucha de esta población vulnerable para satisfacer las necesidades de su supervivencia.

Una ilustración de las características que acompañan cada uno de los indicadores de presión y estado, ayudará a la comprensión de los posibles componentes de MST (Respuesta), así como los impactos que éstos pudieran ocasionar. El Cuadro 6 muestra la aplicación del componente de degradación de suelos en la zona de estudio.

Dentro de las principales causas que amenazan a las sabanas orientales venezolanas destacan: la presión antropogénica sobre los recursos forestales (tala clandestina o extracción de madera para la elaboración de productos maderables y no maderables) y la práctica de actividades como la quema. Esto ha ocasionado un desequilibrio ambiental, poniendo en peligro la supervivencia de las poblaciones animales y vegetales que habitan este patrimonio ecológico. Sin embargo, los procesos asociados con la dinámica de los cambios ocurridos en la cobertura vegetal y uso del suelo, son algunos de los principales factores que determinan la permanencia, disminución y extinción de los ecosistemas de sabanas en el oriente venezolano (Cuadro 7).

Es evidente que, durante las últimas décadas, las actividades antrópicas se han convertido en el principal desencadenador de las transformaciones 
Cuadro 6. Aplicación del componente de degradación de suelos.

\begin{tabular}{clll}
\hline Nivel & $\begin{array}{l}\text { Problema } \\
\text { ambiental }\end{array}$ & \multicolumn{1}{c}{ Tipo de Indicador } & \multicolumn{1}{c}{ Característica } \\
\hline & & $\begin{array}{l}\text { Presión } \\
\text { (fuerza causante) }\end{array}$ & Monocultivo, sobreexplotación \\
\cline { 3 - 4 } & $\begin{array}{l}\text { Estado } \\
\text { (condición resultante) }\end{array}$ & $\begin{array}{l}\text { Degradación química y física, Dismi- } \\
\text { nución de la fertilidad, descenso de los } \\
\text { rendimientos }\end{array}$ \\
\cline { 3 - 4 } & Suelos & $\begin{array}{l}\text { Respuesta } \\
\text { (acción mitigante) }\end{array}$ & $\begin{array}{l}\text { Cambio de uso de tierra hacia policul- } \\
\text { tivos; aplicación de materia orgánica, } \\
\text { agricultura de conservación }\end{array}$ \\
\cline { 3 - 4 } & degradados & $\begin{array}{l}\text { Disminución del proceso de degrada- } \\
\text { ción; incremento de los rendimientos } \\
\text { en un 5 \% anual e incrementada la } \\
\text { disponibilidad y diversidad de produc- } \\
\text { tos agrícolas }\end{array}$ \\
\hline
\end{tabular}

Fuente: adaptado de Urquiza et al. (2011).

de los ecosistemas terrestres, por encima de los fenómenos naturales, en el caso particular de Kashaama, Estos procesos de transformación o cambio que experimentan las coberturas vegetales y usos del suelo del territorio, son considerados en este estudio como una de las principales causas que coadyuvan al deterioro ambiental y en consecuencia influyen directamente en la calidad de vida de los habitantes de la zona (Olivares et al., 2017b). El Cuadro 8 establece los aspectos claves para la aplicación del componente relacionado con la baja calidad de vida.

Cuadro 7. Aplicación del componente de poca cubierta vegetal.

\begin{tabular}{llll}
\hline Nivel & $\begin{array}{c}\text { Problema } \\
\text { ambiental }\end{array}$ & \multicolumn{1}{c}{ Tipo de Indicador } & \multicolumn{1}{c}{ Característica } \\
\hline & & $\begin{array}{l}\text { Presión } \\
\text { (fuerza causante) }\end{array}$ & $\begin{array}{l}\text { Monocultivo para grandes extensiones de } \\
\text { terreno }\end{array}$ \\
\cline { 3 - 4 } Local $\begin{array}{c}\text { Poca cubierta } \\
\text { vegetal }\end{array}$ & \begin{tabular}{l} 
Incendios forestales en época seca y tala no \\
(condición resultante) \\
\cline { 3 - 4 }
\end{tabular} & $\begin{array}{l}\text { Respuesta } \\
\text { controlada }\end{array}$ \\
\cline { 3 - 4 } & (acción mitigante) & $\begin{array}{l}\text { Implementación de sistemas de vigilancia } \\
\text { comunitaria con prevención y combate }\end{array}$ \\
\hline & Impacto & $\begin{array}{l}\text { Incremento de la capacidad de captura de } \\
\text { carbono de la atmosfera y la disminución } \\
\text { de la superficie afectada por incendios }\end{array}$ \\
\hline
\end{tabular}

Fuente: adaptado de Urquiza et al. (2011). 
Barlin Orlando Olivares-Campos - Miguel Armando López-Beltrán - Deyanira Lobo-Luján Changes in land use and vegetation in the agrarian community Kashaama, Anzoátegui, Venezuela: 2001-2013

Cuadro 8. Aplicación del componente de baja calidad de vida en la comunidad indígena Kashaama.

\begin{tabular}{llll}
\hline Nivel & $\begin{array}{l}\text { Problema } \\
\text { ambiental }\end{array}$ & \multicolumn{1}{c}{ Tipo de Indicador } & \multicolumn{1}{c}{ Característica } \\
\hline & & $\begin{array}{l}\text { Presión } \\
\text { (fuerza causante) }\end{array}$ & Poca disponibilidad de alimentos \\
\cline { 3 - 3 } & & $\begin{array}{l}\text { Estado } \\
\text { (condición resultante) }\end{array}$ & $\begin{array}{l}\text { Insuficiente ingestión de calorías/ } \\
\text { persona/día }\end{array}$ \\
\cline { 3 - 4 } Local & 22Baja cali- & $\begin{array}{l}\text { Respuesta } \\
\text { (acción mitigante) }\end{array}$ & $\begin{array}{l}\text { Incorporación de tierras ociosas a } \\
\text { ciclo productivo, rotación de cultivos } \\
\text { y fortalecimiento de la agricultura } \\
\text { familiar }\end{array}$ \\
\cline { 3 - 4 } & & $\begin{array}{l}\text { Incremento de la capacidad de captura } \\
\text { de carbono de la atmosfera y la dis- } \\
\text { minución de la superficie afectada por } \\
\text { (Efecto }\end{array}$ & $\begin{array}{l}\text { incendios } \\
\text { transformador). }\end{array}$ \\
& &
\end{tabular}

Fuente: adaptado de Urquiza et al. (2011).

\section{Conclusiones}

La degradación de tierras en la región provoca que los agricultores de la comunidad indígena estudiada, que se quedan desarrollando apenas la agricultura de subsistencia frente en predios agropecuarios degradados, se enfrenten la carencia de alimentos a mediano plazo.

Esta problemática se ha visto reflejada en la mayoría de las tierras del municipio Freites, debido, principalmente, a las operaciones de exploración y producción de crudos de las empresas petroleras que funcionan a lo largo del municipio, desafectando cientos de hectáreas y causando daños serios al medio ambiente, en la mayoría de los casos irrecuperables.

La dinámica espacio temporal del uso de suelo y vegetación en Kashaama se define en base a la permanencia de la cobertura, la totalidad (100\%) del asentamiento e infraestructura se encuentran constantes, mientras que aproximadamente el $90 \%$ de los cultivos tradicionales de la zona permanecen; $80 \%$ de la vegetación natural que se ubica en algunas zonas presenta continuidad, mientras que la vegetación secundaria persiste en menor proporción.

Finalmente, la comunidad indígena estudiada, presenta un proceso significativo de transformación de la cobertura vegetal a usos del suelo 
antrópicos, poniendo en riesgo los bienes y servicios ecosistémicos para la población.

La comunidad agrícola indígena de Kashaama vive en un sector que presenta una problemática representada principalmente por la contaminación de los recursos naturales, el deterioro de los servicios públicos y el abuso económico respecto de los recursos naturales que ofrece la tierra. El abuso de la explotación de los recursos naturales ha ocasionado migraciones internas, extrema pobreza, discriminación y exclusión de los ciudadanos indígenas. La falta de recursos o apoyo financiero para ejecutar sus labores agrícolas es también una de las causas de los principales problemas sociales representados en la comunidad, a quienes, por falta de recursos económicos, se les ha negado la oportunidad de obtener orientaciones educativas.

En este sentido, es lógico pensar que la degradación del suelo y los problemas ambientales asociados a la contaminación del agua y suelos por la actividad petrolera del municipio repercuten directa o indirectamente en los factores de cambios culturales asociados a la subsistencia de la etnia indígena.

La agricultura, en esta comunidad indígena Kari'ña, es valorada por la dinámica compleja de las relaciones agroproductivas y socioeconómicas cotidianas, así como por su función transcendental en la producción de alimentos y diversos servicios ecosistémicos de interés común. Los participantes de esta investigación lograron identificar problemas que no pueden solucionar por sí mismos y reconocen la necesidad de recurrir al poder institucional para hallar posibles soluciones, promoviendo y consolidando así la integración entre diferentes grupos de la comunidad, el trabajo conjunto con instituciones externas a ella y la participación de todos los grupos comunitarios, incluidos adultos mayores, mujeres y niños.

\section{Referencias}

Banco Mundial. (2008). Manejo sostenible de la tierra: desafíos, posibilidades y costos de oportunidad. Bogotá, Colombia: Banco Mundial y Mayol Ediciones S.A.

Colditz, R. R., Llamas, R. M. \& Ressl, R. A. (2014a). Detecting change areas in Mexico between 2005 and 2010 using 250 m MODIS images. IEEE Journal on Selected Topics in Applied Earth Observation and Remote Sensing, 7 (8), 3358-3372. 
Colditz, R. R., Pouliot, D., Llamas R. M., Homer, C., Latifovic, R., Ressl. R.A., Meneses Tovar, C., Victoria Hernandez, A. \& Richardson, K. (2014b). North American-wide land cover change detection between 2005 and 2010 with 250 m MODIS data. Photogrammetric Engineering \& Remote Sensing, 80 (10), 918-924.

Comerma, J. (2002). Capacidad de uso, aptitudes y vocación de uso de las tierras. Caracas, Venezuela: Instituto Nacional de Tierras.

Comerma, J. \& Chacón, E. (2002). Aptitud de los llanos venezolanos para los principales usos ganaderos. XVIII Cursillo sobre Bovinos de Carne. R. Romero, J. Arango y J. Salomón (Eds.) Maracay: Facultad de Ciencias Veterinarias, UCV. 2002: 193-216. Impreso

Comerma, J. \& Paredes, R. (1978). Principales limitaciones y potencial agrícola de las tierras en Venezuela. Agronomía Tropical, 28(2), 71-85. Recuperado el 14 de febrero de 2015, de http://sian.inia. gob.ve/repositorio/revistas_ci/Agronomia\%20Tropical/at2802/arti/ comerma_j.htm

Delgado, F. (2007). Propuesta metodológica para evaluar y asignar la vocación de uso a las tierras rurales. Caracas, Venezuela: Instituto Nacional de Tierras.

Ferrer, J. \& Comerma, J. (1997). Riesgos de erosión hídrica bajo el uso actual de la tierra en las mesas orientales de Venezuela. Venesuelos, $5(1$ y 2$), 1-8$.

Millennium Ecosystem Assessment. (2005a). Ecosystems and Human Well-being: Synthesis. Washington, DC: Island Press.

Millennium Ecosystem Assessment. (2005b). Ecosystems and Human Well-being: Desertification Synthesis. Washington, DC: World Resources Institute.

Organization for Economic Co-operation and Development (OECD). (1993). Environmental Indicators: Basic Concepts and Terminology. Background paper No. 1. París: OECD.

FAO (Organización de las Naciones Unidas para la Alimentación y la Agricultura). (1990). Evaluación de tierras para la agricultura de regadío: Directivas. Boletín de Suelos (55). Roma, Italia: FAO.

FAO (1996). Forest resources assessment 1990. Survey of tropical forest cover and study of change processes. Roma, Italia: FAO. 
FAO. (2008). Climate Change and Food Security: A Framework Document. Recuperado el 7 de febrero de 2016, de: http://www.fao.org/ forestry/15538-079b31d45081fe9c3dbc6ff34de4807e4.pdf

FAO/LADA. (2009). Manual de Evaluación Local de la Degradación de Tierras Áridas (LADA-L). Roma, Italia: FAO.

Foley, J. A., DeFries, R., Asner, G. P., Barford, C., Bonan, G., Carpenter, S. R., Chapin, F.S., Coe, M.T., Daily, G.C., Gibbs, H.K., Helkowski, J.H., Holloway, T., Howard, E.A., Kucharik, C. J., Monfreda, C., Patz, J. A., Prentice, I. C., Ramankutty, N. \& Snyder, P. K. (2005). Global consequences of land use, Science, 309, 570-574.

García-Mora, T. J. \& Mas, J. F. (2011a). Modland: los productos de superficie terrestre MODIS. En J.F. Mas (Comp.). Aplicaciones del sensor MODIS para el monitoreo del territorio $(25$ - 70). Instituto Nacional de Ecología (INE), Secretaría de Medio Ambiente y Recursos Naturales (SEMARNAT), Universidad Nacional Autónoma de México (UNAM), Centro de Investigaciones en Geografía Ambiental (CIGA).

García-Mora, T.J. \& Mas, J.F. (2011b). Evaluación de imágenes del sensor MODIS para la cartografía de la cobertura del suelo en una región altamente diversa de México. Boletín de la Sociedad Geológica Mexicana, 63(1), 83-94.

Land Processes Distributed Active Archive Center (LP DAAC). (2014). Land Cover Type Yearly L3 Global 500 m SIN Grid. Recuperado el 25 de enero de 2016, de https://pdaac.usgs.gov/dataset_discovery/ modis/modis_products_table/mcd12q1

Lobo D., Cortez, A., Rodríguez, M. F., Ovalles, F., Rey, J. C., Gabriels, D. \& Parra, R. M. (2010). Análisis de la agresividad y concentración de las precipitaciones en Venezuela. I. Región de los llanos. Bioagro, 22(3), 169-176.

Lozano, Z., Lobo, D. \& Pla, S. I. (2002). Susceptibilidad a la degradación física de alfisoles de los llanos centrales y occidentales de Venezuela. Rev. Fac. Agron, 28,41-57.

Mogollón, L.F. \& Comerma, J. (1994). Suelos de Venezuela. Caracas: Editorial Exlibris. 
Olivares B., Rodríguez, M., Cortez, A., Rey, J.C. \& Lobo, D. (2015). Caracterización físico natural de la comunidad indígena de Kashaama con fines de manejo sostenible de la tierra. Acta Nova, 7 (2),141-162 Olivares, B. (2012a). Características agroclimáticas de la Mesa de Guanipa y requerimientos de clima y suelo de la planta de soya. En: M.E. Martin, A.V. Parra y M. González (coord.). El cultivo de la Soya en la Mesa de Guanipa ( 05-21).Caracas: MAT Editorial.

Olivares, B. (2012b). Valorización del conocimiento ancestral y local mediante la percepción del clima en comunidades agrícolas indígenas del Sur de Anzoátegui, Venezuela. Revista UDO Agrícola. 12 (2):407-417.

Olivares, B. (2014). Relación de la naturaleza, el clima y la espiritualidad de las comunidades indígenas agrícolas Kari'ña del estado Anzoátegui, Venezuela. Revista Tiempo y Espacio. 61 (2), 129-150.

Olivares, B. Parra, R. y Cortez, A. (2017c). Characterization of precipitation patterns in Anzoátegui state, Venezuela. Ería. 3 (3), 353-365.

Olivares, B. y Cortez, A. (2017). La extensión agrícola en territorios indígenas Kari'ña de Venezuela: Hacia el desarrollo local sostenible con identidad. Saarbrücken, Alemania, Editorial Académica Española..

Olivares, B. y Franco, E. (2015). Diagnostico agrosocial de la comunidad indígena de Kashaama: Un estudio empírico en el estado Anzoátegui, Venezuela. Revista Cientifica Guillermo de Ockham. 13 (1), 87-95.

Olivares, B., Cortez, A., Muñetones, A. y Casana, S. (2016a). Strategic Elements of Organizational Knowledge Management for Innovation. Case: Agrometeorology Network. Revista Digital de Investigación en Docencia Universitaria, 10(1), 68-81.

Olivares, B., Cortez, A., Parra, R., Lobo, D., Rodríguez, M.F y Rey, J.C. (2017a). Evaluation of agricultural vulnerability to drought weather in different locations of Venezuela. Rev. Fac. Agron. (LUZ), 34 (1), 103-129.

Olivares, B., Guevara, E. y Demey, J. (2012). Utilización de bioindicadores climáticos en sistemas de producción agrícola del estado Anzoátegui, Venezuela. Revista Multiciencias. 12 (2), 136-145.

Olivares, B., Guevara, E., Oliveros, Y. y López, L. (2013a). Aplicación del índice de confort térmico como estimador del estrés calórico en 
la producción pecuaria de la Mesa de Guanipa, estado Anzoátegui. Revista Zootecnia Tropical. 31 (3), 209-223.

Olivares, B., Lobo, D., Cortez, A., Rodríguez, M.F. y Rey, J.C. (2017b). Socio-economic characteristics and methods of agricultural production of indigenous community Kashaama, Anzoategui, Venezuela. Rev. Fac. Agron. (LUZ), 34(2), 187-215.

Olivares, B., Torrealba, J. y Caraballo, L. (2013b). Variability of the precipitation regime in the period 1990-2009 in the location of El Tigre, Anzoátegui state, Venezuela. Rev. Fac. Agron. (LUZ), 30(1), 19-32.

Olivares, B., Zingaretti, M.L., Demey Zambrano, J.A. y Demey, J.R. (2016b). Tipificación de los sistemas de producción agrícola y la percepción de la variabilidad climática en Anzoátegui, Venezuela. Revista FAVE - Ciencias Agrarias, 15(2), 39-50.

Rodríguez, M.F., Cortez, A., Olivares, B., Rey, J.C, Parra, R. y Lobo, D. (2013). Análisis espacio temporal de la precipitación del estado Anzoátegui y sus alrededores. Agronomía Tropical, 63 (1-2), 57-65.

Rodríguez, M.F. \& Rey, J.C. (2004). Delimitación de zonas frágiles de Venezuela. Mimeo. Maracay: INIA-CENIAP.

Rojas, T. (1981). Geografía de la región nororiental. Caracas, Venezuela: Editorial Ariel- Seix Barral Venezolana

Salas M., Delgado, F., Esteva, Y. \& Sampson, M. (2008). La ordenación del territorio y la vocación de uso agrícola de la tierra en Venezuela. Revista Geográfica Venezolana, 49(2), 267-288.

Sulla-Menashe, D \& Friedl, M. A. (2018). User Guide to Collection 6 MODIS Land Cover (MCD12Q1 and MCD12C1) product. Recuperado: 20 de noviembre de 2018. https://pdaac.usgs.gov/sites/default/files/ public/product_documentation/mcd12_user_guide_v6.pdf

Urquiza, N. M., Alemán, C., Flores, L., Ricardo, M., Aguilar, Y. (2011). Manual de procedimientos para manejo sostenible de tierras. Cuba: Editorial CIGEA.

Zinck A. \& Urriola, P.L. (1970). Origen y evolución de la Formación Mesa: un enfoque edafológico. ler. Congreso Venezolano de la Ciencia del Suelo. Maracaibo. Sociedad Venezolana de la Ciencia del Suelo. (53-63). 